\title{
I FÓRUM DE INTEGRAÇÃO DOS SERVIÇOS DE SAÚDE E DAS ATIVIDADES DE ENSINO, PESQUISA E EXTENSÃO DA USP
}

\author{
$1^{\text {ST FORUM ON INTEGRATION OF HEALTH SERVICES WITH USP }}$ \\ TEACHING, LEARNING, RESEARCH AND COMMUNITY ACTIVITIES \\ *Namie Okino Sawada, **Vinicius Pedrazzi, ***Maria de Lourdes Veronese Rodrigues
}

\section{RESUMO}

O campus de Ribeirão Preto da Universidade de São Paulo engloba cinco unidades que prestam serviços de saúde no município de Ribeirão Preto e região. As crescentes demandas e atividades influenciam na provisão de serviços de acordo com as políticas públicas de saúde e motivaram a idealização e realização do I Fórum de Integração dos Serviços de Saúde e das Atividades de Ensino, Pesquisa e Extensão da USP.

O evento teve a finalidade de discutir as relações da universidade com os serviços de saúde, as fontes de fomento e programas do Ministério da Saúde. Após receberem informações contidas nas diversas palestras ministradas no primeiro dia do Fórum, os participantes foram distribuídos em cinco grupos multidisciplinares e multiprofissionais de discussão sob o tema: "Inserção, infraestrutura, recursos humanos e materiais e interdisciplinaridade nos serviços de saúde".

Neste artigo, os autores resumem os principais tópicos discutidos e apresentam as sugestões elaboradas pelos grupos de discussão.

Palavras-chave: Serviços de saúde. Universidade. Políticas públicas de saúde.

\section{ABSTRACT}

The campus of Ribeirão Preto, University of São Paulo, with its five Health Sciences units, provides health services in the county of Ribeirão Preto and region. The increasing demands and activities have influence in provision of services according to of public health policies and led to the idealization and realization of the $\mathrm{I}^{\text {st }}$ Forum on Integration of Health Services with USP Teaching, Learning, Research and Community Activities.

The aim of the meeting was to discuss the relationship between the university and the health system, the sources of financial support and programs of the Ministry of Health. The second day, after the first day attending lectures, the participants were distributed into five multidisciplinary and multiprofessional discussion groups about the theme: "Activities, infrastructure, human and materials resources and interdisciplinarity in the provision of health services".

In this paper, the authors summarize the main topics discussed and report the suggestions elaborated by the discussion groups.

Key words: Health services. University. Public health policies.

\footnotetext{
* Docente do Departamento de Enfermagem Geral e Especializada da Escola de Enfermagem de Ribeirão Preto da Universidade de São Paulo (EERP-USP) e presidente da Comissão de Cultura e Extensão Universitária (CGEx) da EERP-USP. ** Docente do Departamento de Materiais Dentários e Prótese da Faculdade de Odontologia de Ribeirão Preto (FORP-USP) e presidente da CCEx da FORP-USP. **** Docente do Departamento de Oftalmologia, Otorrinolaringologia e Cirurgia de Cabeça e Pescoço da Faculdade de Medicina de Ribeirão Preto (FMRP-USP) e presidente da CCEx da FMRP-USP. Comissão de Cultura e Extensão Universitária da Faculdade de Medicina de Ribeirão Preto - USP - Av. Bandeirantes, 3.900 - I4048-900 - Ribeirão Preto - SP - e-mail: mdlvrodr@fmrp.usp.br.
} 


\section{INTRODUÇÃO}

As crescentes demandas e atividades que repercutem no cotidiano do funcionamento para o atendimento das políticas públicas de saúde e que envolvem os alunos de graduação e pós-graduação (stricto e lato sensu), bem como os docentes das respectivas unidades, foram fundamentais para a idealização e realização desse encontro, no campus de Ribeirão Preto, onde cinco unidades distintas têm envolvimento direto no ensino, pesquisa e extensão, com práticas de saúde para a população da cidade e da região.

\section{MATERIAL E MÉTODO}

O I Fórum de Integração dos Serviços de Saúde e das Atividades de Ensino, Pesquisa e Extensão da USP foi realizado no Bloco Didático da Faculdade de Medicina de Ribeirão Preto da USP nos dias I5 e I6 de março de 20II. O evento foi aberto pelo diretor da FMRP-USP, o Prof. Dr. Benedito Carlos Maciel; pela coordenadora, a Profa. Dra. Namie Okino Sawada; pela diretora da EERP-USP, a Profa. Dra. Silvia Helena De Bortoli Cassiani e pelo pró-reitor adjunto de Extensão Universitária, o Prof. Dr. José Ricardo de Carvalho Mesquita Ayres, que deram as boasvindas. Também compôs a mesa diretora o diretor da FORP-USP, o Prof. Dr. Osvaldo Luiz Bezzon.

A seguir, houve palestras ministradas pelo superintendente do Hospital das Clínicas de Ribeirão Preto, o Prof. Dr. Marcos Felipe Silva de Sá, pelo secretário municipal de saúde de Ribeirão Preto, o doutor. Stênio José Correia Miranda, e pelo pró-reitor adjunto de Extensão Universitária, Prof. Dr. José Ricardo de Garvalho Mesquita Ayres.

Esse primeiro fórum teve a finalidade de discutir as relações da universidade com os serviços de saúde, as fontes de fomento e programas do Ministério da Saúde.

Foram discutidos os seguintes tópicos: as relações interinstitucionais, a comunicação acadêmica e da rede municipal de saúde; as atenções primária, secundária e terciária à saúde; o papel dos preceptores; os recursos disponíveis em termos de parque de equipamentos e instrumentos instalados, os serviços oferecidos, os recursos humanos disponíveis e as carências; o papel da academia e da municipalidade, bem como dos governos federal e estadual nos respectivos âmbitos do Ministério da Saúde e da Secretaria Estadual da Saúde. Discutiu-se inclusive o papel do ensino, da pesquisa e da extensão, que tem diferentes entendimentos entre as partes ouvidas.

A comunicação entre o usuário dos serviços (cliente), os preceptores, os agentes de saúde disponíveis na rede de atenção, aqueles concursados e estáveis, os residentes e aprimorandos e seu importantíssimo papel na atenção secundária e terciária à saúde também foram exaustivamente discutidos.

Após a primeira mesa estabelecida e discutida na manhã do dia I5, houve nova mesa de apresentação e discussão à tarde, com a exposição do Programa PET-Saúde pela Profa. Dra. Maria do Carmo G. G. Caccia-Bava da Faculdade de Medicina de Ribeirão Preto da USP. A professora historiou o programa e apresentou a atuação das unidades de ensino-saúde do campus de Ribeirão Preto, com a necessária interdisciplinaridade em harmônico funcionamento, apresentando em vídeo os resultados concretos e confirmando a importância do envolvimento dos alunos de graduação em Medicina, Enfermagem e Odontologia, sob constante orientação docente, na atenção básica à saúde e na difusão dos princípios que norteiam o SUS. Também sinalizou alguns problemas levantados quanto à execução do programa, como a vulnerabilidade social e violência urbana (queixa e problema a ser enfrentado pelos alunos de graduação); salientou-se ainda a resistência dos docentes quanto à mudança da prática acadêmica (deslocamento do habitat de trabalho para atuar nos locais onde a atenção básica à saúde é oferecida).

$\mathrm{Na}$ apresentação do PET-Saúde Mental pela Profa. Dra. Lucilene Cardoso da Escola de Enfermagem de Ribeirão Preto da USP, destacou-se a importância de se estabelecerem programas especiais do PET-Saúde para atender necessidades prementes e com crescente demanda na atenção aos usuários de álcool e drogas. Por se tratar da primeira versão do programa, tem como objetivo mapear os locais e as necessidades do serviço de saúde mental, objetivando o fortalecimento do processo de integração da universidade com o serviço de saúde, bem como proporcionar educação permanente a partir de um projeto políticopedagógico embasado na ética.

A residência profissional e multiprofissional foi abordada pelo Prof. Dr. Cássio Edgard Sverzut da Faculdade de Odontologia de Ribeirão Preto da USP. O professor reportou a dificuldade em montar um curso de residência na FORP, informando inclusive que havia dois regimentos distintos na unidade tratando da residência odontológica; um dentre eles, feito à imagem 
daquele da residência médica, obrigava a percepção de bolsa devido ao compromisso de atuação em dedicação integral. A captação de bolsas foi uma árdua batalha, ainda não resolvida pela falta de editais lançados pelos governos nas diferentes esferas de atuação pública. Ressaltou a importância do serviço de extensão prestado pelos residentes (não bolsistas), com alta qualificação, e que não é suprido na graduação devido à complexidade dos traumatismos que frequentemente chegam à universidade. Informou que, apesar de os residentes ainda não serem remunerados, há uma crescente procura pela atividade de residência na unidade, demonstrando a carência e a importância desse tipo de formação. A presença dos residentes no serviço de saúde, além de ser um facilitador da relação ensino e serviço, proporciona atendimento de alta complexidade, atendendo a uma demanda da sociedade que não encontra similaridade nos serviços públicos de saúde instalados.

Para o segundo dia do fórum, I6 de março, foram estabelecidos cinco grupos de discussão sob o tema "Inserção, infraestrutura, recursos humanos e materiais e interdisciplinaridade nos serviços de saúde”. Os grupos, caracterizados pela multidisciplinaridade de atuação em saúde, eram constituídos por graduandos, docentes, residentes da USP e servidores da rede municipal de saúde, além de profissionais em saúde da rede privada.

\section{RESULTADOS}

\section{GRUPO I}

No Grupo I, coordenado pela Profa. Dra. Maria da Glória Chiarello de Mattos da Faculdade de Odontologia de Ribeirão Preto da USP, uma série de pontos foi levantada, apresentada a seguir.

I) É importante a inserção da universidade no serviço de saúde para a visão da atenção primária, secundária e terciária pelos alunos.

2) $\mathrm{O}$ atendimento de pacientes é agendado pelo serviço (regulador) que muitas vezes fica aquém das necessidades do ensino.

3) A universidade tem mentalidade voltada para o ensino; o serviço de saúde é direcionado para o número de atendimentos. Para melhor conciliar ensino e serviço é necessário aumentar o número de recursos humanos.

4) A presença do aluno de graduação no serviço é uma forma de desenvolver o senso crítico e, deste modo, fazer a diferença na formação do profissional da saúde.

5) O aluno no serviço pode contribuir com a educação continuada do profissional da equipe, pois acrescenta novas ideias e conhecimentos.

6) É importante que os docentes conheçam a realidade dos serviços de saúde e busquem interagir na construção da atuação universidade-serviço para melhor planejamento, evitando assim número excessivo de alunos de diferentes áreas da saúde, o que dificulta a atuação e, consequentemente, o aprendizado.

7) É importante o desenvolvimento dos diferentes níveis do aprendizado, como o aperfeiçoamento e residência junto à pós-graduação em serviços de saúde, para o atendimento aos diferentes níveis de complexidade, suprindo uma alta demanda dos serviços e a qualificação na formação desses profissionais. Ressalta-se que a sua presença se dá para a integração entre os diferentes profissionais da saúde e não apenas para a substituição de profissionais.

8) É imperativo valorizar o profissional em particular e a equipe em serviço: docente, profissional e agente de saúde.

9) Há de se ter políticas governamentais de fomento para recursos humanos e infraestrutura - como, por exemplo, Pró-Saúde e PET-Saúde - mas que se tornem programas e não projetos, deixando de sofrer limitação temporal.

IO) É imprescindível educar a população quanto ao SUS, quanto às profissões e o que as diferentes áreas da saúde executam. Por exemplo, o Conselho de Fisioterapia divulgará informativo sobre o papel do fisioterapeuta e as diferentes modalidades ou áreas de atuação, uma vez que há muita desinformação sobre o papel do profissional da área.

II) Educar os agentes comunitários (educação continuada) para melhor formação profissional e melhor atuação como cuidadores atuando na equipe.

I2) Promover cada vez mais reuniões (simpósios multiprofissionais) para melhorar a interação entre os diversos profissionais da saúde e, assim, melhorar a atuação em equipe.

I3) Quanto à infraestrutura, uma pergunta fica no ar: a quem cabe a responsabilidade por ela? O grupo discutiu e concluiu que a todos os envolvidos no processo ensino-saúde: 
- Governo Federal - programas SUS.

- Governo Estadual - programas SUS.

- Governo Municipal - programas SUS.

- Universidade - programas SUS.

\section{GRUPO II}

O Grupo II, coordenado pelo Prof. Dr. Cássio Edvard Sverzut da Faculdade de Odontologia de Ribeirão Preto da USP, discutiu e ponderou duas subáreas em específico, tal como se segue.

No tocante à graduação:

a) Os alunos deveriam ser inseridos no sistema de saúde pública, porém com adequados incentivo e avaliação.

b) Constatou-se que existe uma grande resistência de alguns docentes e alunos em realizar atividades extramuros, por fatores diversos.

Com relação aos problemas da residência multiprofissional:

a) Constatou-se a falta de comunicação e articulação entre os setores envolvidos (áreas da saúde). Muitas vezes ocorre um desconhecimento da própria instituição do serviço e do que ele pode oferecer.

b) A universidade precisa disponibilizar meios para acompanhar, avaliar e auxiliar a inserção do aluno no programa. Nem todas as áreas têm docentes ou supervisores.

c) Falta de infraestrutura para acomodar todos os integrantes do programa.

d) É primordial a questão do incentivo financeiro. $\mathrm{O}$ oferecimento de bolsas é importante e deve ser almejado.

e) $\mathrm{O}$ ensino não deve ser compartimentalizado, já que é um programa multiprofissional. O programa deve proporcionar uma integração entre os profissionais, resultando, por exemplo, em discussão conjunta de casos clínicos. Em ensino multiprofissional deve necessariamente existir transdisciplinaridade.

f) A atuação dos residentes se faz necessária e essencial não apenas no sentido de viabilizar o serviço, mas também em propor melhorias, aprimorando, assim, os trabalhos do grupo e, consequentemente, o atendimento ao cliente.

g) Melhorar a comunicação entre os membros do grupo como, por exemplo, no preenchimento de prontuários clínicos.

h) Há uma preocupação latente com o "dia seguinte" à formação, ou seja, o mercado de trabalho. Constitui preocupação dos residentes em serem absorvidos pelo mercado de trabalho após o término da residência.

i) A residência multiprofissional é um acontecimento relativamente novo e, portanto, está passando por um processo de conhecimento dos profissionais da área de saúde, das próprias instituições e da população em geral. Nesse sentido, é importante que os integrantes deste programa atuem como agentes multiplicadores de conhecimento, oferecendo, por exemplo, palestras e simpósios. Novamente, é fundamental o papel do residente.

\section{GRUPO III}

O Grupo III teve como coordenadora a Profa. Dra. Silvia Matumoto da Escola de Enfermagem de Ribeirão Preto da USP e centrou a discussão em três assuntos principais, a seguir.

Com relação à formação de Equipe Saúde da Família (ESF) em Ribeirão Preto:

a) Nota-se a falta de abrangência e de investimento na formação e ampliação do número. No Espírito Santo, o Programa Saúde da Família (PSF) abrange $100 \%$ da população.

b) No âmbito da universidade, é preocupante a disputa de modelos - especialista $\mathrm{x}$ generalista -, com mudanças de estruturas curriculares que nem sempre são reflexos das necessidades da sociedade.

c) Há uma falta de conhecimento da população a respeito dos serviços prestados e onde buscá-los. Sugere-se maior e mais eficaz divulgação.

d) Ainda existe uma dificuldade em aceitar o modelo da atenção básica. Os clientes solicitam um especialista.

e) $\mathrm{O}$ atendimento primário tem que trabalhar muito bem para filtrar os casos que vão para o secundário e o terciário.

f) Muitas vezes, por conta do pronto atendimento, a população desconhece os benefícios que os outros profissionais da área de saúde podem oferecer.

g) Algumas incongruências ainda são notadas, como, por exemplo, o limite geográfico. São atendidas somente as famílias que estão na área de abrangência do núcleo, contradizendo o princípio de universalidade pressuposto pelo próprio SUS. 
Para a relação ensino-serviço:

a) Existem dificuldades da formação. O tema saúde pública - SUS - é abordado de forma pouco atrativa na teoria.

b) Muitos cursos têm o contato com a prática somente no último ano. Como sugestão para a resolução do problema, talvez haja necessidade de mudanças da grade curricular.

c) O Programa PET-Saúde é pouco divulgado.

A respeito da residência multiprofissional:

a) Foi gerada uma proposta de maior carga horária na ESF.

b) Sente-se que houve uma dificuldade no início, a saber, resistência na recepção da equipe para se adequar aos horários, além de falha na interlocução entre preceptores e serviços. Como solução apontada, a resistência seria revertida a partir de um bom trabalho em equipe.

c) A residência favorece a multidisciplinaridade e a criação de maior vínculo com a população e com a equipe (dois anos).

d) Outra dificuldade apontada foi a carga horária, que desfavorece a elaboração do Trabalho de Conclusão de Curso (TCG). Como sugestão, deverse-ia intentar a criação de um espaço-horário.

e) Foi também sinalizado o atraso no pagamento das bolsas.

f) Não se deve aceitar que os governos em suas respectivas instâncias entendam a residência como uma mão de obra barata! O governo deveria investir mais nisso!

\section{GRUPO IV}

O Grupo IV, coordenado pelas profas. doutoras Janete Cinira Bregagnolo, da Faculdade de Odontologia de Ribeirão Preto da USP, e Maria José Bistafa, da Escola de Enfermagem de Ribeirão Preto da USP, buscou possíveis soluções para os vários problemas apontados.

I) Todos os docentes da universidade deveriam passar pelas unidades básicas de saúde para conhecer o que acontece e o que se passa realmente neste setor diminuindo o preconceito e a falta de diálogo do sistema.

2) Os preceptores e as estruturas deveriam dispor de instrumentos adequados para os níveis de instrução, objetivando uma atenção de ótima qualidade.

3) Dever-se-ia criar um fórum para discutir e definir a hierarquização adequada do sistema de saúde a fim de saber articular os níveis de atenção à saúde.

4) Uma importante meta a ser atingida é a valorização das atividades docentes que integram as atividades de extensão com ensino e pesquisa, bem como daquelas de outros profissionais que atuam na comunidade.

5) É preciso potencializar a integração multiprofissional a fim de aperfeiçoar o serviço e o atendimento ao público.

6) Também se deve incentivar a continuidade e integração entre os docentes das áreas básicas com as especializadas.

7) A saúde da comunidade deve ser obrigatória em todos os cursos, incluindo estágios in loco.

8) Para evitar que o professor que atua na comunidade para Ensino Básico seja desvalorizado, sugerese trabalhar e modificar a forma de pontuação e avaliação curricular, considerando os anos trabalhados e as atividades de extensão e não somente valorizar as instituições de fomento à pesquisa.

9) É necessário melhorar a integração com as secretarias municipais e estaduais de saúde para que os projetos e melhorias sejam resolvidos da melhor maneira possível, apontando as dificuldades e auxiliando a integração entre serviço e universidade.

IO) Os participantes concluíram a discussão com a seguinte assertiva: a comunicação entre educação e informação para o cliente é essencial!

\section{GRUPO V}

Coube à Profa. Dra. Marlívia Gonçalves Carvalho Watanabe da Faculdade de Odontologia de Ribeirão Preto da USP a coordenação do Grupo $V$, que procurou trabalhar a inserção.

I) Criação de comissão intra-campus para melhoria da inserção de outras especialidades além da Medicina (tradição). Nesse sentido foi proposta a criação de um grupo multidisciplinar de gestão no campus.

2) Melhor estruturação da forma de inserção do profissional no serviço de saúde.

3) Contrapartidas pactuadas. Ainda há distorções entre o que é proposto e o que é oferecido.

4) A falta de profissionais na rede para recepcionar os alunos ainda é um problema. Como solucionar? 
5) Deve haver um reforço da Comissão junto aos diretores de unidade e pró-reitores sobre a importância da integração entre os serviços de saúde e a academia no seu tripé de funções precípuas.

6) Melhor discussão com os profissionais do serviço sobre a entrada de estagiários, residentes, etc., a fim de orientar melhor os benefícios para o serviço.

7) Deve-se investir muito nos programas de capacitação - educação continuada para os funcionários (desenvolvimento do lado pedagógico) da instituição (investimento da universidade).

8) Procurar reverter em benefícios como férias, especialização, extensão.

9) Solicitação de verbas para contratação do funcionário (preceptor).

IO) A equipe entende o papel dos estagiários e residentes.

II) Com relação ao PET-Saúde, este oferece benefícios para os profissionais que recebem os alunos, mas não deveria ser apenas para alunos graduados e não graduados, como também técnicos de Enfermagem, farmácia, assistente social, ou muitas vezes ficam mais com os alunos que os graduados.

12) Valorizar profissionais que não possuem titulação em nível superior.

I3) Identificar as reais necessidades dos serviços para inserir alunos.

I4) Criação de falsa demanda (continuidade dos serviços).

I5) Necessidade de melhor estruturação curricular dos cursos de graduação.

I6) Aproximar pesquisa em serviço e revertê-las em prol dos serviços (infraestrutura), como exemplo, buscar apoio em agências estatais de fomento;

I7) Redirecionamento de verbas (processos de trabalho, materiais), como exemplo, sabe-se que a mesma verba que vem para Odontologia, vem para o Direito, e o curso de saúde tem maior custo.

I8) Deve-se incentivar e praticar a interdisciplinaridade desde a graduação:

- promover atendimento integral;

- disciplina interunidades (mudança de cultura).

I9) A residência multiprofissional (interdisciplinaridade, supervisão, planejamento, teoria, publicação) possibilita a integração, o planejamento e a cooperação.

20) Valorização das atividades de assistência exercida pelos docentes (não há publicação Qualis A de experiências) e um estímulo à aproximação dos docentes.

2I) Reforçar junto à administração da USP sobre a importância da assistência na formação da área da saúde (criar parâmetros específicos de avaliação).

\section{DISCUSSÃO}

O Sistema Único de Saúde (SUS) é reconhecido como o maior plano de saúde do mundo, e é público. Apesar desse reconhecimento, é sabido que existem problemas, seja em termos de formação e distribuição de equipes de saúde, seja na distribuição e repasse de fomentos (recursos financeiros), o que muitas vezes é entrave para o bom funcionamento do sistema. O SUS deve integrar os hospitais universitários e as redes de saúde, demandando a coordenação conjunta de metas e serviços, com a necessária integração dos currículos dos cursos de graduação, atendendo também à LDB, que prevê a graduação em saúde com atuação interdisciplinar, visando à saúde holística do cliente.

Entretanto, é notório que o ensino de graduação não consegue suprir as crescentes demandas dos setores secundário e terciário, e a necessidade de mão de obra especializada para essas modalidades de atendimento em saúde. No fórum, o assunto foi discutido com muita ênfase e sugere-se que essas demandas podem ser supridas perfeitamente com a formação lato sensu de programas de residência, residência multiprofissional e cursos de especialização, de aperfeiçoamento e outros.

O papel da academia (universidade) nos serviços de saúde, antes visto como meramente de ensino e pesquisa, hoje necessariamente deve contemplar atividades de extensão com objetivos e compromissos de transformação social.

Essa nova filosofia requer a valorização das atividades de cultura e extensão nos âmbitos governamentais e acadêmicos. Infelizmente, nota-se ainda uma dificuldade na interlocução entre as partes, pois nem sempre a visão e as necessidades de uma são pactuadas com aquelas da outra. Para uma maior eficácia do atendimento SUS, seria fundamental que o ensino fosse integrado às necessidades dos serviços de saúde.

Fundamental para que essas metas sejam atingidas seria a universidade valorizar as atividades de cultura e extensão, estimulando o docente a se dedicar mais profundamente à formação de recursos humanos qualificados para atender às demandas por serviços de 
saúde pela sociedade, uma vez que atualmente lhe é cobrado maciçamente sua produção intelectual (papers) em detrimento de outras atividades-fins que compõem o tripé da universidade.

No campus de Ribeirão Preto, a integração acadêmica das unidades nos serviços de saúde se encontra ainda em diferentes estágios. Enquanto que Medicina e Enfermagem são tradicionalmente integradas aos serviços de atendimento e gerenciamento de saúde, Odontologia, Farmácia e Psicologia ainda encontram dificuldades patentes nessa integração, necessitando de mais e maiores espaços de formação acadêmica conjunta, integrando disciplinas e recursos humanos como facilitadores na formação de equipes de saúde.

No âmbito da unidade FMRP, o curso de Medicina, instalado em 1952, e posteriormente com a fundação da EERP em I96I, houve uma necessária inserção do trabalho em equipe entre as duas unidades. Mais recentemente, com a criação dos cursos de Fisioterapia, Terapia Ocupacional, Nutrição e Metabolismo, Fonoaudiologia e Informática Biomédica na FMRP, a multidisciplinaridade na atenção ao cliente foi consolidada. Entretanto, essa inserção ainda carece de aperfeiçoamento.

Uma importante estratégia de integração dos conteúdos multidisciplinares veste-se na residência multiprofissional, que agrega ainda a Farmácia e a Psicologia; porém, a captação de recursos para a concessão de bolsas ainda é difícil e insuficiente. Mesmo no caso das bolsas concedidas, há relatos recorrentes de atrasos no pagamento e regularidade das bolsas. Algumas unidades, como a Odontologia, têm programas de residência profissional funcionando há dois anos e não conseguiram sequer bolsas para os residentes, mesmo a demanda pelo serviço sendo grande e crescente (traumatismos orofaciais) e a procura pela residência ser igualmente importante por parte dos profissionais.

Ressalta-se ainda a importância da inserção dos residentes nos serviços de saúde, oferecendo notável qualificação no atendimento secundário e terciário, bem como a manutenção e atualização dos campos de estágio, o que proporciona um melhor ensino aos alunos de graduação. Assim, nota-se que o residente atua inclusive como forte e presente elo de união entre os preceptores e graduandos, oferecendo continuidade na assistência e contribuindo para a qualidade dos serviços.

\section{COMENTÁRIOS FINAIS E CONCLUSÕES DO FÓRUM}

Frente a todas as discussões desenvolvidas nesse I Fórum, alguns pontos foram comuns aos grupos, como:

I) Promover integração entre as diferentes áreas de atuação em serviços de saúde, visando à valorização holística da saúde e dos profissionais que a ela se dedicam.

2) Fica evidente a necessidade de formar um grupo coordenador intra-campus para discutir as questões de inserção das unidades nos serviços de saúde bem como, discutir a residência multiprofissional no âmbito do campus de Ribeirão Preto, esse grupo teria a função de agir como um facilitador dessa integração entre a academia nos seus três eixos-mãe (ensino, pesquisa e extensão) e os serviços de saúde.

3) Os participantes foram unânimes quanto à necessidade de serem realizadas novas edições desse Fórum, para que se possa discutir, com conhecimento de causa, a relação entre as demandas de atendimento em saúde pelas diversas secretarias de saúde em níveis governamentais e o que a academia pode e deve oferecer, preparando-se adequadamente para tal, com reais incentivos para os preceptores, aprimorandos e equipes envolvidas.

\section{EQUIPES PARTICIPANTES}

\author{
GRUPO I \\ - João Paulo de Medeiros \\ - Adriana A.B. Murashima \\ - Letícia Oliveira Neri da Silva \\ - Vânia Aguiar \\ - Milena Amorim \\ - Régia Maria G.R. Sobral \\ - Bruna Cristina Carponas \\ - Paula Tapia Gomes Pereira \\ - Cintia Megumi Yagui \\ - Fernanda C. Zanardo Cecília Prado Sales da Silva \\ - Milena Junqueira Reis \\ - Camila Polisello \\ - Telma Kioko Takeshita \\ - Stephanie Robinson \\ - Ana Paula F. Chediek
}


GRUPO II

- Maurício Serra Ribeiro

- Sara Galleni de Oliveira

- Rosa Aparecida Oficiati Macedo

- Taiuani Marquine Raymundo

- Priscila Galo Farnocchi

- Paula dos Santos Pereira

- Letícia Caroline Doretto

- Maria Eugênia Silvestre e Silva

- Mariela H. Bisson

- Fernanda Bergamini

- Lorena Borges

GRUPO III

- Carina Porfírio Miranda

- Marília Braz U. Dias

- Lívia Pimenta Bonifácio

- Daniela dos Santos

- Priscila Villa

- Mariana Silva Matos

- Denise Mayumi Tanaka

GRUPO IV

- Priscila Maria Manzini Ramos

- Renata Martins Campos Gramado

- Maria de Lourdes V. Rodrigues

- Gustavo Santos Mercedes

- Fernando Grivelenti Vilar

- Hélio Fugishima

- Vanessa Wagner

- Giovanni Trentin Ferronato

- Danielly Oliveira Silva

\section{GRUPOV}

- Alessandra Nagano Gavallari

- Felipe Dias Carvalho

- Fernando Santa Cecília Artuzo

- Cristiane Soncino Silva

- Alexandre Laguna Terreri

- Juliana Maria de Paula

- Vanice Soares Lopes

- Maria Rita Lerri

- Gleice Cristina Colombari

- Thais Sêneda de Mecenas

- Jaime Augusto Gerveira

- Karina Carolina Macedo Alves 